Schulze u. Urich: Ueb. d. Zusammensetzung etc. 321.

\title{
Ueber die Zusammensetzung des Wollfetts.
}

\author{
(Zweite Abhandlung) \\ von \\ Ernst Sohulze und A, Urioh. \\ (Ref. E. Schulze.)
}

Durch eine früher in diesem Journal [2] 7, 163 xeröffentlichte Arbeit hat der Eine von uns nachgewiesen, dass im Wollfett nehen freiem Cholesterin zusammengesetzte Aether des Cholesterins und eines zweiten mit dem Cholesterin isomeren Alkohols, des lsooholestrins, sich tinden.

Die Frage nach der Zusammensetzung des Wollfetts konnte jedoch auf Grund der bei dieser Arbeit erhaltenen Resultate nicht ganz vollständig besntwp ortet werden. Ans der Elementarzusammensetzung des rohen Gemenges der Wollfett-Alkohole ergab sich, dass in demselben neben Cholesterin und Isocholesterin noch ein Kohlenstoff-ärmerer Alkohol (oder eine Anzahl von solchen) vorkommen müsse; über die Beschaffenheit dieses Koblenstoff-ärmeren Alkobols konnten aber noch gar kejne Angaben gemacht werden. Ferner schien sus dem Mengenverhältniss, in welchem. Wollfett-Alkohole und Säuren bei Zerlegung des in Weingoist schwer löslichen Wollfett-Theils erhalten wurden, hervorzugehen, dass im Wollfett auch freie Fettsäuren sich finden; doch konnte dieser Schluss nicht als zweifollos hingeatellt werden.

Zur Entacheidung dieser Fragen haben wir nach Beschaffung neuen Materials die Untersuchung fortgesetzt. Zu dieser Fortsetzung wurden wir ferner auch bewogen durch den Wunsch prüfen za können, ob die in dem früber untersuchten Wollfett anfgefundenen Bestandtheile auch in andern Wollfett-Sorten sich finden. Denn da das Fett verschiedener Woll-Sorten in seinen äusseren Eigenschaften oft beträchtliche Verschiedenheiten zeigt, so wäre Joum. f. prakt. Chemio [8] Ba. 9. 
es möglich, dass auch in der chemischen Zusammensetzung beträchtliche Unterschiede stattfinden.

$\mathrm{Zu}$ den im Folgenden mitgetheilten Untersuchungen haben wir vorzugsweise eine Fett-Sorte benutzt, welche aus der Rohwolle norddeutscher Landschafe durch Extraction mit Aether dargestellt war (diese Wolle enthält in lufttrocknem Zustande 7\% Fett). Das so gewonnene Fett haben wir zur Unterscheidung von der früher untersuchten Fett-Sorte (a) mit b bezeichnet. Einige Bestimmungen sind ferner auch ausgeführt worden mit einer Fett-Sorte wus der Rohwolle eines schweizerischen Bergschafs (im Folgenden mit c bezeichnet). ${ }^{1}$ )

Die Zerlegung des Wollfetts wurde ganz wie früher ansgefiihrt. Dasselbe wurde zunächst in einen in Weingeist leicht löslichen und einen darin schwer löslichen Theil gespalten, indem man es wiederholt mit Weingeist auskochte und die entstandenen Lösungen nach dem Erkalten von der ungelöst gebliebenen Fettmasse abgoss. Beim Erkalten der Lösungen schieden sich weisse Flocken aus denselben aus, welche lür sich gesammelt wurden. Die Menge derselben war, besonders beim Wollfett $b$, nicht ganz unbedeutend, aber doch nicht gross genug, um sie bei der weiteren Behandlung getrennt halten zu können; sie wurden daher mit der ungelöst gebliebenen Fettmasse vereinigt.

Beim Wollfett $\mathbf{b}$ wurde diese Zerlegung quantitativ durchgeführt. Bei 8 maligem Ausziehen mit Weingeist wurden aus demselben annähernd 32 p.C. aufgelöst. Doch zeigte sich, dass in der so erhaltenen Lösung neben eigentlichen Wollfett-Bestandtheilen sehr bedeutende Mengen von Seifen (ohne Zweifel ölsaures Kalium) enthalten waren. Auf das stetige Vorkommen dieser Verbindungen in dem durch Extraction der Rohwolle mit Aether dargestellten Fett ist bereits früher aufmerksam gemacht worden. ${ }^{2}$ )

1) Beide Fett-Sorten können nach der bei den Landwirthen üblichen Bezeichnungøweise zum loichtflüssigen Wollfett gorechnet werden.

2) Vgl. dies Journ. 108, 194. 
Man kann diese Verbindungen entfernen, indem man das rohe Fett in Aether löst und die Lösung mit Wasser durchschüttelt; sie werden dann vom Wasser aufgenommen. Ein Versuch, diese Trennung in diesem Falle quantitativ durchzuführen, misslang jedoch; beim Durchschütteln der ätherischen Fettlösung mit Wasser entstand eine Emulsion, welche sich nach wochenlangem Kochen nicht vollständig wieder trennte. Annähernd lässt sich jedoch die Menge der dem Rohfett beigemischten ölsauren Salze auch aus der Aschenmenge berechnen, welche beim Verbrennen des Fetts zurückbleibt und welche fast ausschliesslich aus kohlensaurem Kalium besteht.

Der in Weingeist leicht lösliche Theil vom Wollfett b hinterliess 9,1 p.C. Asche; nimmt man an, dass diese Asche ausschliesslich aus $\mathrm{KCO}_{3}$ bestanden hat und dass dem Fett nur ölsaures Kalium beigemischt war, so berechnet sich daraus für den genannten Wollfett-Theil ein Gehalt von 50,7 p.C. an ölsaurem Kalinm; nur 49,3 p.C. dieses Wollfett-Theils wüden demnach aus eigentlichen Fettbestandtheilen bestanden haben. Das reine, frei von ölsauren Salzen gedachte Wollfett würde also enthalten haben

$$
\begin{aligned}
& 18,9 \text { p.C. in Weingeist leicht lösliche Substanz } \\
& \frac{81,1}{100,0} " \text { " " }
\end{aligned}
$$

Selbstverständlich ist die Trennung mittelst Weingeists keine scharfe; die zurückbleibunde Fettmasse ist ja im Weingeist nicht unlöslich, sondern nur schwerlöslich

Jeder der so erhaltenen Wollfett-Theile wurde nun mit alkoholischer Kalilauge verseift. Die entstandenen Lösungen wurden im Wasserbade eingedampft, die Rückstände mit Wasser angerïhrt und mit Aether geschüttelt. Die Wollfeti-Alkohole wurden vom Aether aufgenommen, während die Kaliseifen in die wässrige Lösung übergingen ${ }^{1}$.

1) Um die Wollfett-Alkohole vollständig zu gewinnen, ist es nöthig, das Durchschütteln öfter und mit stets erneuerten Mengen von Aether 
Der in Weingeist leicbb lösliche Theil vom Wollfett $b$ und $c$ lieferte, eben so wie der gleiche Theil des frïher untersuchten Wollfetts, bei dieser Zerlegung bedeatende Mengen ron Cholesterin, welches durch Krystallisation aus Aether-Weingeist leicht rein zu exhalten war. Es liess auch hier wieder nachweisen, dass der grösste Theil dieses Cholesterins nicht erst durch die Verseifung aus zusammengesetzten $\lambda$ ethern abgeschieden, sondern schon ursprünglich in freiem Zustande vorhanden gewesen war. Denn als einer nicht verseiften Parthie dieses WollfettTheiles durch Wasser die beigemengten ölsauren Salze zum allergrössten Theil entzogen worden waren, krystallisirte aus der weingeistigen Lösung des Restes Chole. sterin in bedentender Menge und ganz ohne Beimengung von flockiger Substanz (freies Isocholesterin konnte also hier nicht oder doch wenigstens nur in höchst geringer Menge vorhauden sein). ${ }^{1}$ )

Der in Alkohol schwerlösliche Theil des Wollfetts b lieferte bei der Zerlegung mit alkoholischer Kalilauge neben Kaliseifen ein Gemenge von Wollfett-Alkoholen, welches in seinem Ansehen und in seinen Eigenschaften ganz mit dem Product übereinstimmte, welches in gleicher

zu wiederbolen; denn es scheint, dass ein kleiner Theil der ersteren in der wässrigen Seifenlösung sich auflöst und von derselben nur silmählich an den Aether abgegeben wird. Fs ist ferner za bemerken, dass die beim Durchschütteln der Seifenlösnngen mit Aether sich bildenden Emulsionen sich zuwoilen schon nach einigen Stunden, zuweilen aber erst nach mehreren Tagen in die ätherisehe und wässrige Schicht trennen. Durch diese Umstände wird die von uns zur Trennnng der Wollfott-Alkohole und Seiten angewendete Methode zu einer ziemlioh schwierigen; sie hat aber wenigstens den Vortheil, dass sie die WollfettAlkohole vollkommen frui von Seifen liefert.

1) Doch war auch in diesem Wollfett-Theil ein Theil der Alkohole in Form zusammengenetzter Aether vorhanden. Denn bei Zerlegung einer von ölsauren Salzen fast vollständig befreiten Parthie desselben wurden 9,4 Grm. Wollfett-Alkohole und 3,4 Grm. Bäuren erhalten; die letzteren schienen vorzugsweise aus Oelsäure zu bestehen. Auch flüchtige Fettsäuren, auf deren Vorkommen schon in der friheren Abhandlong aufmorksam gemacht ist, waren hior vorhanden, aber nar in sehr yeringer Menge. 
Weise aus dem früher untersuchten Wollfett erhalien worden war.') Aus einer Lösung dieses Gemenges in beissem Weingeist sehieden sich beim Erkalten weisse, amorphe Massen ans, welche die Gegenwart von Isot cholesterin andeuteten. Zur Trennung desselben von dem gleichzeitig vorhandenen Cholesterin wurde wieder die früher beschriebene Methode angewendet: die WollfettAlkohole wurden in die Benzoësäure-Aether übergeführt und die ätherische Lösung der letzteren der langsamen Verdunstung überlassen. Neben den tafelförmigen $\mathbf{K}_{\mathbf{r y}}$ stallen des Benzoësäure-Cholesterin-Aethers schied sich der Benzoësäure-Isocholesteriu-Aether in feinen Krystallnadeln aus, welche durch Schlämmen getrennt werden konnten. Das aus letzterem abgeschiedene Isocholesterin zeigte die früher angegebenen Eigenschaften. Die Elementaranalyse einer Probe ergab einen Kohlenstoffgehalt von 83,44 p.C., einen Wasserstoffgehult von 11,86 p.C. (während die Formel $\mathrm{C}_{25} \mathrm{H}_{44} \mathrm{O} 83,87$ p.C. $\mathrm{C}$ und 11,83 p.C. $\mathrm{H}$ verlangt). Der Schmelzpunkt einer aus wiederholt umkrystallisirtem Benzoësäure-Aether dargestellten und daher als $r$ in anzusehenden Portion lag bei $138^{\circ}$, stimmte also mit dem. friiher gefundenen Schmelzpunkt (137-138 $)$ uberein. $\left.{ }^{2}\right)$

Das Isocholesterin schien im Wollfett $b$, ebenso wie in dem früher untersuchten Wollfett, in annähernd eben so grosser Menge vorhanden zu sein, als das Cholesterin.

1) Fine Flementaranalyse crgab für dasselbe einen Gehalt von 82,0 p.C. $C$ und 12,4 p.C. $H$.

2) Bs ist vielleicht nicht überflüssig, darauf aufmerksam zu machen, dass die von uns gemachte Annahme, dass der als Isocholesterin bezeichnete Alkohol isomer mit dem Cholesterin ist, als eine zweifellos bewiesene nicht betrachtet werden kann, insofern bei so hochstomigen Vorbindungen die Resultate der Analyse stets einen gewissen Spielraum für die Wahl der Formel lassen. Es würden z. B. Verbindungen von den Formeln $\mathrm{C}_{25} \mathrm{H}_{42} \mathrm{O}$ oder $\mathrm{C}_{27} \mathrm{H}_{46} \mathrm{O}$ (Homologe des Cholesterins) in ihrer procent. Zusammensetzung vom Cholesterin so wenig abweichen, dass die Diftereuzen innerhalb der Feblergrenzen der Analyse liegen. Du indessen die früher nitgetbeilten, bei dex Analyse des Isocholesterins und seiner Verbindungen erbaltenen Zahlen oben so 
Anders dagegen war es beim Wollfett c. Auch hier fand sich Isocholesterin, aber nur in geringer Menge im Vergleich zum Cholesterin. Als daher das aus dem schwer löslichen Wollfett-Theil abgeschiedene rohe Gemenge der Wollfett-Alkohole in viel heissem Weingeist gelöst wurde, krystallisirte beim Erkalten anfangs nur Cholesterin ohne Beimischung von Isocholesterin; esst aus der Mutterlauge schied sich auch letzteres aus.

Unser Hauptaugenmerk richteten wir auf die Gewinnung des neben Cholesterin und Isocholesterin noch vorhandenen Kohlenstoff-ärmeren Alkohols. Aus den Resultaten der früheren Arbeit schien sich zu ergeben, dass derselbe nur in geringer Menge im Wollfett vorhanden sei; denn es war nicht möglich gewesen, aus dem durch Erhitzen der Wollfett-Alkohole mit Benzoësäure dargestellten Gemenge der Benzoësäure-Aether kei der früher beschriebenen Trennung durch Krystallisation aus ätherischer Lösung ausser Benzoësäure-Cholesterin-Aether und Benzoësäure - Isocholesterin-Aether noch ein drittes Product in einer für nähere Untersuchung genügenden Menge zu isoliren. Es wurde indessen schon in der früheren Mittheilung darauf aufmerksam gemacht, dass eine in etwas grösserer Menge vorhandene dritte Aether-Art dann der Beoachtung entgehen konnte, wenn sie in Weingeist löslich war. Denn das Gemenge der Benzoësäure-Aether wurde, bevor man die Zerlegung desselben durch Krystallisation aus ätherischer Lösung vornahm, mit Weingeist ausgekocht, um etwa vorhandenes freies Cholesterin und Isocholesterin zu entfernen. Diese Bebandlung erschien nöthig, weil nach den von Berthelot gemachten Angaben nicht zu erwarten war, dass beim Erhitzen des Cholesterins mit Benzoësäure eine ganz vollständige Ueberführung desselben in Benzoësäure-Aether erfolgte. Mit dem unverbunden gebliebenen Cholesterin und Isocholesterin konnte

gut auf die Formel $\mathrm{C}_{28} \mathrm{H}_{44} \mathrm{O}$ passen, als die Analysen, aus denen man für das Cholesterin diese Formel abgeleitet hat, so ist man wohl berechtigt, auch dem Isocholesterin diese Formel zu geben. 
zugleich die dritte Aether-Art durch den Weingeist gelöst worden $\left.\operatorname{sein}^{1}\right)$.

Es kam daher darauf an, für die Darstellung der Benzoësäure-Aether eine Methode anzuwenden, bei der man sicher sein konnte, die Wollfett-Alkohole vollstän dig in die Benzoësäure-Aether überzuführen. Wir haben, um dies zu erreichen, die genannten Alkohole mit Benzo ësüure-Anhydrid zusammen geschmolzen $\left.{ }^{2}\right)$. Auch in diesem Falle geht die Aetherbildung nicht etwa momentan, sondern nur allmählich vor sich; wir haben daher die geschmolzene Masse 48 Stunden lang (auf ca. $180^{\circ}$ ) erhitzt.

Nach Beendigung des Erhitzens wurde die Reactionsmasse unter Zusatz von etwas Weingeist in einem Mörser fein zerrieben, sodann zur Entfernung der gebildeten Benzoësäure und des überschüssigen Benzoësäure-Anhydrids zuerst mit einer kalten Lösung von kohlensaurem Natrium, darauf mit warmem Wasser behandelt. Der Rückstand, welcher nun die gesammten Wollfett-Alkohole als BenzoylVerbindungen enthalten musste, wurde getrocknet.

10 Theile Wollfett-Alkohole lieferten so 12,64 Theile Benzoësäure-Aether. Es ist das fast genau die Menge von Benzoësäure-Aethern, welche hätte entstehen müssen, wenn das Gemenge der Wollfett-Alkohole nur aus Cholesterin und Isocholesterin bestände. Die genaue Uebereinstimmung kann zufällig sein; denn es war kaum möglich, bei den oben beschriebenen Operationen Substanzverluste ganz zu vermeiden; doch geht aus diesen Zahlen wobl schon hervor, dass der neben Cholesterin und Isocholesterin vorhandene kolllenstoffärmere Alkohol annähernd eben so viel Benzoyl zu seiner Ueberführung in

1) Diese entstandenen stark braun gefärbten Lösungen wurden nicht weiter untersucht, da eine I'rennung eines in denselben etwa vorhandenen Benzoësëure-Aethers von dem gleichzeitig vorhandenen freien Cholesterin nicht möglich erschien.

2) Auf 1 Theil Wollfett-Alkohol wurde 1/2 Theil Benzoësäure-Anhydrid angewendet. 
Benzoësäureăther beansprucht, als Cholesterin, also ein annähernd eben so hohes Aequivalentgewicht besitzen muss.

Eine abgewogene Probe des rohen Qemenges des Benzoëstăure-Aethers wnrde nun mit einer kleinen Menge von Weingeist ausgekocht, sodann auf ein Filter gebracht und längere Zeit mit kaltem Weingeist ausgewaschen, darauf wieder getrocknet und gewogen. Fs zeigte sich, dass durch den Weingeist etwa 20 p.C. der angewendeten Substanz aufgelb̈st worden wareu. Aus der durch Bindampfen concentrirten J aus, welche auch nach völligem Erkalten weich und unkrystalliniseh blieben. Sie lösten sich schwer in kaltem, leichter in kochendem Weingeist, sehr leicht in Aether. Die nähere Untersuchung ergab, dass sie ans einem Benzoesäure-Aether bestanden, der in seinen Eigenschaften vom Benzoësăure-Cholegterin-Aether nnd vom Benzoësäure-Isocholesterin-Aether ganz verschieden war.

Da diese Verbindung sich in kaltem Aether ausserordentlich leicht auflöst, so lässt sie sich auch durch dieses Lösungsmittel bis zu einem gewissen Grade von den Benzoësäure Aethorn des Cholesterins und des Isocholesterins trennen, welche beide in kaltem Aether ziemlich schwer löslich sind (1 Theil Benzoësäure-Cholesterin-Aether bedarf bei $15^{\circ}$ ungefähr 36 Theile Aether znr Lösung; 1 I'h. Benzoësäure-Isocholesterin-Aether ungefähr $70 \mathrm{Th}$.). Zu diesem Zweck wurde das rohe Gemenge der Benzoësäure-Aether mit wenig kaltem Aether behandelt. Aus der so entstandenen, ziemlich stark braun gefärbten Lösung $\left.{ }^{1}\right)$ liessen wir durch Verdunsten bis auf ein geringeres Volum die mitgelösten Benzoësäure-Aether des Cholesterins und Isocholesterins zum grössten Theile sich ausscheiden; die letzte Mutterlauge lieferte dann den dritten Aether in amorphem Zustande, gemengt mit verhältnissuäseig

2) Das zarückbleibende Gemenge der Benzoësäare-Acther des Cholesterins und Isocholesterins war diggegen fast farblos. 
geringen Mengen der beiden vorher genannten Aetherarten. Um letztere so weit sls möglich zu entfernen, zogen wir noch einmal mit kaltem Aceton ans; anch in diesem Lösuugsmittel läst sich der amorphe Aether sehr leicht, während die Benzoësäure-Aether des Cholesterins und Isocholesterins darin (in der Kälte) sehr schwer löslich sind.

Bei der Zerlegang mit alkoholischer Kalilauge lieferte der amorphe Aather neben benzoësaurem Kalium einen Alkohol, welcher sich in Aether, Aceton und Weingeist schon in der Kälte leicht löste, aber aus keinem der genannten Lösungsmittel krystallisirt zu erhalten war. Derselbe besass eimen schwachen aromatischen Gernch, schmolz schon in gelinder Wärme und liess sich nicht unzersetzt destilliren. Er war nicht ganz frei von Cholesterin za erhalten (gab daher noch schwach die Reaction des Cholesterins mit Schwefelsänre und Chloroform).

Eine Substanz von ganz gleichen äusseren Eigenschaften liess sich auch gewinnen aus dem in Weingeist leicht losslichen Theile des Wollfetts. Sie schien hier zum Theil in Verbindung mit Säuren (vorzugsweise mit Oelsäure), zum Theil vielleicht auch in freiem Zustande vorzukommen.

Die Eigenschaften des so dargestellten Alkohols geben keine Garantie dafür, dase derselbe, auch abgesehen von der Verunreinigung durch Cholesterin, ein chemisch einfacher Körper war; eine Ulementaranalyse desselben kant daher kaum besondern Werth beanspruchen. Wir haben indessen eine solche doch ausgeführt, um zu constatiren, dass hier wirklich eine alkoholartige Substanz vorliegt, welche C-ärmer ist, als Cholesterin. Die Analyse gab folgende Zahlen:

0.2247 Grm. Snbstanz (gewonnen aus dem in Weingeist leicht lösliohen Wollfeti, Theile) gaben bei der Verbrennung mit Kupferoxyd $0,6608 \mathrm{Grm}$. $\mathrm{CO}_{2}$ and 0,2486 Grm. $\mathrm{H}_{2} \mathrm{O}$, entepreohend 80,14 p. C. C und 12,29 p. C. H.

Die Zerlegung des Benzoêsäure-Aethers dieser Substanz wurde quantitativ susgeführt: 
8,16 Grm. desselben gaben dabei 5,883 Grm. des Alkohols (=72,1 p.C. voin Gewicht des Benzoësäure-Aethers) ${ }^{\mathfrak{}}$ ).

Was die Quantität betrifft, in welcher dieser amorphe Alkohol in dem in Weingeist schwer löslichen WollfettTheile sich findet, so scheint aus den früher mitgetheilten Zahlen hervorzugehen, dass mindestens 20 p.C. von dem rohen Gemenge der Benzoësäure-Aether aus dem Benzoësäure-Aether dieses Alkohols bestanden; denn so viel löste sich in Weingeist. Allerdings war bei der Behandlung mit Weingeist ohne $Z$ weifel auch etwas von den andern Benzoësäure-Aethern aufgelöst worden, welche ja in Weingeist nicht ganz unlöslich sind; andrerseits aber ist es unwahrscheinlich, dass der amorphe Aether durch das Ausziehen mit Weingeist ganz vollständig gewonnen wurde, $d a$ er sich $j a$ in diesem Lösungsmittel nur ziemlich schwer löst.

Die Quantität, in welcher der amorphe Alkohol in dem in Weingeist leicht löslichen Wollfett-Theile sich findet, ergiebt sich annähernd aus folgenden Notizen: $42 \mathrm{Grm}$. des aus diesem Wollfett-Theile abgeschiedenen Gemenges der Wollfett-Alkohole lieferten durch Krystallisation aus Aether-Weingeist zunächst ungefähr $10 \mathrm{Grm}$. fast völlig reines Cholesterin. Aus der davon abfiltrirten Lösung schied sich nach dem Verdunsten auf ein geringeres Volum noch eine bedeutende Menge von unreinem, mit Isocholesterin gemengten Cholesterin aus. Es blieb schliesslich eine ziemlich dicke Mutterlauge, aus welcher direkt kein Cholesterin mehr zu gewinnen war. Beim Eindampfen liess dieselbe etwa $20 \mathrm{Grm}$. Rückstand; dieser Rückstand bestand vorzugsweise aus dem amorphen Alkohol. Er wurde rait Benzoësäure-Anhydrid zusammengeschmolzen. Aus dem so dargestellten Gemenge dér Benzoësäure-Aether liesseu sich noch einige Gramme des Cholesterin- und des

1) Zur Vergleichung führen wir an, dass Benzoësäure-CholesterinAether bei der Zerlegung $\mathbf{7 8 , 2}$ p.C. seines Gewichts an Cholesterin liefert. 
Isocholesterin-Aethers abscheiden '); der Rest bestand aus dem amorphen Benzoësäure-Aether.

Aus den vorstehenden Zahlen ergiebt sich, dass in dem neu untersuchten Wollfett (b) der amorphe Aether in ziemlioh bedeutender Menge sich vorfand. Es ist wohl zu vermuthen, dass das Gleiche auch in dem früher untersuchten Wollfett der Fall war, dass der amorphe Alkohol aber hier wegen des bei der Untersuchung eingeschlagenen Ganges (und wegen unvollständiger Untersuchung des in Weingeist leicht löslichen Wollfett-Theils) der Beobachtung eńtging. ${ }^{2}$ )

Ueber die Säuren des Wollfetts sind, wie in der früheren Mittheilung erwähnt ist, schon von Anderen einige Angaben gemacht worden; Reich und Ulbricht haben nachgewiesen, dass unter ihnen Stearinsäure und Oelsäure sich finden; eine vollständige Untersuchung derselben aber liegt bis jetzt nicht vor. Auch wir konnten eine solche bei den von uns untersuchten Wollfett-Sorten nicht ausführen, da die daraus gewonnene Quantität an festen Fettsäuren dazu nicht hinreichte. Die Zusammensetzung des Gemenges der letzteren erwies sich ferner als so complicirt, dass eine Zerlegung in die einzelnen Fettsäuren nicht nur sehr viel Material, sondern auch einen sehr beleutenden Arbeitsaufwand beansprucht haben

1) Es zeigte sich hier wieder, dass zur Abscheidung des Cholesterins aus einem Gemenge, aus welchem es durch direkte Krystallisation nicht zu gewinnen ist, die Verwandlung desselben in den Benzoësäure-Aether ein sehr geeignetes Mittel ist; diese Verbindung lässt sich wegen ihrer Schwerlöslichkeit und wegen ihrer grossen Krystallisationsfähigkeit leicht rein erhalten.

2) Es ist möglich, dass ausser Cholesterin, Isocholesterin und dem amorphen Alkohol noch andere alkoholartige Körper in geringur Menge im Wollfett sich finden, denn bei den Methoden, welche zur Trenuung der drei vorgenannten Substanzen angewendet werden mussten, int es kaum möglich, Beimisohungen aufzufinden, welche in geringer Menge vorhanden sind. 
würde. Wir beschränken uns daher auf die folgenden Mittheilungen :

Die aue den Kaliseifen vermittelst verdünnter Scbwefelsâure abgeschiedenen Säuren wurden zunächst in die Bleisalze verwandelt und letztere mit Aether extrahirt; eine bedeutende Menge Bleisalz ging dabei in Lösung. Die daraus abgeschiedene, stark braun gefürbte Sänre verhielt sich wio unreine Oelsäure. Eine Reindarstellung derselben wurde nicht ausgefuibrt. Auch liegt es auf der Hand, dass es kaum ein ungünstigeres Material für die Reindarstellung von Oelsäure geben kann, als das Wollfett. Denn bei der feinen Vertheilung, in der sich dasselbe in der Wolle befindet, kann leicht eine partielle Veränderung der darin enthaltenen Oleïn-Verbindungen durch den Sauerstoff der Luft erfolgen.

Die vom ölsauren Blei befreiten Bleisalze wurder durch Kochen mit verdünnter Salzsäure zerlegt. Die so abgeschiedenen Fetteäuren erstarrten nach dem Schmelzen zu einer ganz unkrystallinischen Masse. Sie lösten sich ziemlich schwer in heissem Weingeist und schieden sich beim Erkalten in anscheinend unkrystallinischen Flocken und Körnern aus. Proben derselben wurden abfiltrirt, ausgepresst, durch Wiederholung der Operation noch mehr von den leichter löslichen Säuren befreit nnd dann der Flementaranalyso nnterworfen. Dieselbe gab folgende Zahlen:

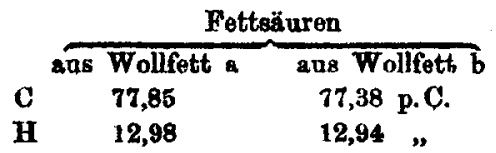

Aus diesen Zahlen ergiebt sich, dass in beiden Wollfett-Sorten Fettä̈uren von höherem Kohlenstoffgehalt, als ihn die Arachinsäure zeigt, rorkommen. Diese Thatsache ist nicht ohne Interesse, $d x$, mit Ausnahme der von Carins entdeckten Hyänasäure so hochatomige Fettsäuren im Körper von Säugethieren in grösserer Menge wenigstens bis jetzt nicht aufgefunden worden sind. 
Die ganze Süure-Masse wurde nun durch fraktionirte Fällung mit Bleiacetat in 6 Portionen zerlegt. Wegen der Schwerlöslichkeit der Süuren in Alkohol konnte die Fraktionirung anfangs nur in der Weise anggeführt werden, dass die h eisse alkoholische Lösung der Säuren mit Bleiacetat versetzt, der entstandene Niederschlag anf einem Wasserbadtrichter heiss abfiltrirt and mit heissem Weingeist ausgewaschen wurde. Die drei letzten Bleiniederschläge entstanden jedoch erst beim Erkalten und warden daher auch erst nach dem Erkalten abfiltrirt.

Die Bleiniederschlägø wurden mit verdünnter Salzsăure zerlegt. Die so gewonnene erste Sâuure-Portion schmolz bei $75-78^{\circ}$, die zweite und dritte bei $66-67^{\circ}$; der Schmelzpunkt der drei letzten lag zwischen 53 und $60^{\circ}$. Keine der so erhaltenen Säure-Portionen konnte als rein angesehen werden. Die erste, am höchsten schmelzende Portion warde durch weitere Fraktionirung in 4 Theile zerlegt. Dieselben zeigten folgende Schmelz - und Erstarrungspunkte:

$\begin{array}{lccc}\text { Schmelzpunkt. } & \text { Erstarrungspunkt. } & \text { Art des Erstarrens. } \\ \text { 1. } & \mathbf{7 8 , 5 - 7 9} & \mathbf{7 7 - 7 7 . 5} & \text { feinkryutallinisch. } \\ \text { 2. } & \mathbf{7 6 - 7 7} & \mathbf{7 4} & \text { deagl. } \\ 3 . & \mathbf{7 6 - 7 7} & \mathbf{7 4} & \text { desgl. } \\ \text { 4. } & \mathbf{7 2 - 7 4} & - & \text { anorph. }\end{array}$

Zu einer weiteren Fraktionirung reichte das Material nicht aus. Proben der zweiten, bei $76-79^{\circ}$ schmelzenden Portion wurden der Elementaranalyse unterworfen. Dieselbe gab folgende Zahlen:

a. 0,8285 Grm. Dubstanz gaben 0,6441 Grm. $\mathrm{CO}_{2}$ and $0,2812 \mathrm{Grm}$. $\mathrm{H}_{2} \mathrm{O}$.

b. 0,2220 Grm. Substanz gaben $0,6432 \mathrm{Grm}$. $\mathrm{CO}_{2}$ und $0,2598 \mathrm{Grm}$. $\mathrm{H}_{\mathbf{2}} \mathrm{O}$.

$\begin{array}{cccc} & 1 . & 2 . & \text { Mittel. } \\ \text { C } & 78,60 & 79.01 & 78,81 \text { p.C. } \\ \text { H } & 12,99 & 13,01 & 13,00 \% "\end{array}$

Diese Zahlen stimmen annähernd uberein mit der Zasammensetzung der $\mathrm{Hyänasäure}=\mathrm{C}_{25} \mathrm{H}_{50} \mathrm{O}_{2}$, welche 78,52 p.C. $C$ und 13,09 p.C. $H$ verlangt, liegen abe aueh nicht weit ab von der Zusammensetzung der Cerotin- 
säure. Der Schmelzpunkt der Hyänasäure wird von Carius zu $77-78^{\circ}$ angegeben, stimmt also ziemlich überein mit dem Schmelzpunkt der untersuchten Säure. Auch die sonstigen Eigenschaften derselben gleichen denen der Hyänasäure; sie löste sich sehr wonig in kaltem, zienlich schwer in heissem Alkohol und schied sich aus dieser Lösung in Körnern aus, welche unter dem Mikroskop als aus feinen Nadeln zusammengesetzt erschienen.

Eine sichere Entscheidung darüber, welche Säure hier vorliegt, ist nicht zu geben, da die Fraktionirung nicht so weit getrieben werden konnte, dass ganz reine Substanzen resultirten. Möglich ist, dass neben Hyänasäure auch die in ihren Eigenschaften und ihrer Zusammensetzung jener sehr nahe stehende Cerotinsäure vorhanden war. Die Entscheidung darüber wird noch erschwert durch den Urnstand, dass der Schmelzpunkt der letzteren nicht genau bekannt ist. Brodie hat fïr Cerotinsäure aus Bienenwachs einen Schmelzpunkt von $\mathbf{7 8}^{\circ}$ angegeben. ) und diese Angabe ist in die meisten Lehrbücher übergegangen; doch erseheint für reine Cerotinsäure dieser Schmelzpunkt zu niedrig, da ja schon die C-ärmere Hyänasäure bei $77-78^{\circ}$ schmilzt; auch giebt Brodie an einer andern Stelle seiner Abhandlung an, dass die dureh Schmelzen von Cerotylalkohol mit Kalihydrat dargestellte Cerotinsäure bei $81^{\circ}$, ,also nur etwa $1^{\circ}$ höher, als Cerotinsäure aus Bienenwachs" schmelze ${ }^{2}$ ).

Diejenigen bei der ersten Fraktionirung erhaltenen Fettsäuren, welche zwischen 53 und $60^{\circ}$ schmolzen, können nach ihrem Schmelzpunkt und ihrem sonstigen Verhalten Gemenge von Stearinsäure und Palmitinsäure gewesen sein; sie lösten sich viel leichter in Weingeist, als die zuerst gefällten Säuren.

Es bleibt uns noch übrig, Angaben über den Gehalt des im Weingeist schwer löslichen Wollfett-Theils an Al-

1) Ann. Chem. Pharm. 67, 187.

ग) A. a. O. S. 208. 
koholen zu machen, um entscheiden zu können, ob dieser Theil nur aus zusammengesetzten Aethern bestand oder anch freie Fettsäuren einschloss. Wir haben diesen Gehalt bei den neu untersuchten Wollfett-Sorten möglichst genau bestimmt. Beim Wollfett $b$ wurden folgende Zahlen erhalten:

a. 11,86 Grm. des in Weingeist schwer löslichen Wollfett-Theils lieferten 6,416 Grm. Wollfett-Alkohole $=54,1$ p.C. vom Gewicht der angewendeten Substanz.

b. 9,15 Grm. derselben Substanz gaben 4,766 Grm. Wollfett-Alkohole $=52,1$ p.C.

Das Mittel aus beiden Bestimmungen ist 53,1 p.C. Diese Zahl spricht für die Annahme, dass der schwer lösliche Theil vom Wollfett $b$ nur aus zusammengesetzten Aethern bestand und keine freien Fettsäuren einschloss. - Denn es wirden z. B. 100 Theile eines im Aequivalentverhältniss zusammengesetzten Gemenges von Stearinsäureund Oelsäure-Cholesterin-Aether und der entsprechenden Isocholesterin-Verbindungen bei der Zerlegung 58,8 Theile Cholesterin und Isocholesterin geben, 100 Theile der analogen Oelsäure- und Hyänasäure-Verbindungen 52,5 Theile Cholesterin und Isocholesterin. Die Gegenwart von Verbindungen des amorphen Alkohols in dem Gemenge wird die procentische Menge an Alkoholen, welche man bei der Zerlegung erhält, nicht viel ändern, da der genannte Alkohol (wie aus der Zusammensetzung seines BenzoësäureAethers zu schliessen ist) fast eben so viel Säure zu binden vermag, als Cholesterin.

Auch lässt sich mit obiger Annahme die Elementarzucammensetzung des schwer löslichen Theils vom Wollfett $b$ in Einklang bringen. Die Analyse gab folgende Zahlen:

$\begin{array}{cccc} & 1 . & 2 . & \text { Mittel. } \\ \text { C } & 81,47 & 81,17 & \mathbf{8 1 , 3 2} \text { p.C. } \\ \text { H } & 12,47 & 12,55 & 12,51 \text { ” }\end{array}$

Es ist das ein C- und H-Gehalt, wie ihn ein Gemenge der Verbindungen des Cholesterins, Isocholesterins und 
des amorphen C-ärmeren Alkohols mit festen Fettsăuren und mit Oelsäure ungefähr besitzen muss.

Anders war es bei dem schwer löslichen Theil von Wollfett e. 11,583 Grm. desselben gaben 5,46 Grm. Wollfatt-Alkohole $=47,1$ p.C. Diese Alkohol-Menge genügt nicht ganz zar Sättigung der vorhandenen Säuren; hier mussten also freie Săuren vorhanden sein, und in der That besass auch der weingeistige Auszug ans diesem Wollfett stark saure Reaction.

Wie in der früheren Abhandlung mitgetheilt worden ist, wurden auch bei der Zerlegung des schwer löslichen Theils rom Wollfett a Alkohole and Säuren in solchem Verhältniss erhalten, dass man das Vorhandensein freier Fettsäuren vermuthen musste; auch die Elementarzusammensetzung dentete darauf hin 1). Diese Annahme konnte jedoch nicht als $z$ weifellos hingestellt werden, weil noch zu erklären blieb, warum diese freien Säuren nicht bei der Behandlung mit Weingeist extrahirt worden waren.

Dies erklärt sich leicht, seitdem nachgewiesen worden ist, dass im Wollfett hochatomige Fettsäuren vorkommen, welche in Weingeist $\mathrm{sch} w e r$ löslich sind. Denn durch die Behandlung mit Weingeist wurde ja das Wollfett nicht in einen in Weingeist löslichen und einen darin unlöslicbon Theil, sondern vielmehr einen in diesem Lösangemittel leicht löslichen und einen darin $\mathrm{schwer}$ löslich en Theil gespalten ${ }^{2}$ ) (die weingeistigen Auszüge wurden erst nach dem Erkalten von der zurückbleibenden Fettmasse abgegossen). Nimmt man an, dass es rorzugsweise oder ausschliesslich die C-reichsten Fettsüuren waren welche in freiem Zustande sich fanden, so ist es nicht auffallend, dass dieselben zum grössten Theil ungelöst zurückblieben; um so mehr, als dieselben durch die gleich-

1) Welche jedoch nicht genau angegeben worden konnte, weil die vorliegenden Analysen sich auf rohes, darch ölzaures Kalium veruereinigtes Fett bezogen.

$\left.{ }^{2}\right)$ Wenn auch in der früheren Abhandlung der in Weingeist sehwer lösliche Wollfałt-Theil der Kürze halber an einigen Stellen sle unlöslicher Theil“" bezeichnet wurde. 
zeitig vorhandenen zusammengesetzten Aether, welche selbst in kochendem Weingeist wenig löslich sind, vor der Einwirkung des Weingeistes mehr oder weniger gesehützt wurden ${ }^{1}$ ). Ein Theil der vorhandenen freien Fettsäuren ist ohne $Z_{w}$ eifel in Lösung gegangen und würde vermuthlich aufgefunden worden sein, wenn der in Weingeist lösliche Theil aus Wollfett a vollständiger untersucht worden wäre ${ }^{2}$ ).

Es ist jedoch noch zu bemerken, dass der Gehalt des Wollfettes a an freien Säuren jedenfalls nicht ganz so gross war, als es nach den in der frïheren Abhandlung angegebenen Zahlen scheint. Dort ist angegeben worden, dass ans dem schwer löslichen Theil dieses Wollfettes auf 1 Theil Wollfett-Alkohole ungefähr 2 Theile Säuren erhalten wurden. Bei näherer Untersuchung dieser Säuren zeigte sich jedoch, dass dieselben noch eine nicht ganz unbeträchtliche Menge von Wollfett-Alkoholen einschlossen (aus $150 \mathrm{Grm}$. konnten noch etwa $10 \mathrm{Grm}$. Alkohole abgeschieden werden). Dieser Gehalt erklärt sich ohne Zweifel daraus, dass bei der früheren Darstellung das Durchschüt-

1) Man könnte sich denken, dass das Gleiche auch für das freie Cholesterin gelten müsse, welches ja auch in kaltem Weingeist schwer löslich ist. Indessen ist es bekannt, dass sich Cholesterin in weingeistigen Lösungen anderer Stofle zuweilen leichter löst, als in reinem Weingeist. So war es auch in diesem Falle. Aus den weingeistigen Auszügen des Wollfettes krystallisirte es direct nicht oder doch nur in höchst geringer Menge aus, obwohl es in bedeutender Menge darin enthalten war; es kxystallisirte aber sofort, nachdem die beigemengten olsauren Salze durch Wasser grösstentheils entfernt worden waren.

9) Der Mangel an Material machte es mir unmöglich, vor Veröffentlichung der früheren Arbeit einige diesen Punkt betreffende Versuche auszuführen, sowie auch das Verhältniss zwischen Alkoholen und Säuren im Wollfett a genauer zu bestimmen. Das Material an Wollfett-Alkoholen, mit welchem ich jene Arbeit ausführte, war schon im Jahre 1870 durch Zerlegung von ungefähr $500 \mathrm{Grm}$. Wollfett dargestellt worden; erst im Winter 1872/73 fand ich Musse, die Arbeit fortzusetzen. $Z u$ dieser Zeit befand sich in meinen Händen nur noch der grösste Theil der aus dem schwer löslichen Wollfett-Theile erhaltenen Alkohole und Säuren, aber gar nichts mehr von unzersetztern Wollfett.

Journal f. prakt. Chemie [2] Bd. 9.

Der Verf. 
teln der Seifen-Lösung mit Aether nicht lange genug fortgesetzt worden ist.

Aus den mitgetheilten Thatsachen geht also hervor, dass die Hauptmasse des Wollfettes aus zusammengesetzten Aethern besteht, dass daneben aber sowohl ein Theil der Alkohole (wenigstens des Cholesterins) als auch zuweilen ein Theil der Säuren in freiem Kustande vorkommt

Einen Gehalt an freien Säuren hat man bekanntlich auch in anderen Fett-Sorten aufgefunden. So findet sich z. B. die Cerotinsäure im Bienenwachs in freiem Zustande; das Palmöl enthält häufig freie Palmitinsằure. Auch im Fett der Samen von Getreide-Arten und Leguminosen ist kürzlich von J. König ${ }^{2}$ ) das Vorkommen freier Fettsäuren nachgewiesen worden.

Ziirich, agrikultur-chem. Laboratorium des Polytechnikums, im März 1874.

\section{Ueber die Dissociation der wasserhaltigen Salze; von}

\section{G. Wiedemann.}

Schon im Jahre 1844 theilt Eilhard Mitscherlich in der 4. Auflage seines Lehrbuchs der Chemie S. 565 folgende Beobachtung mit: „Bringt man Krystalle von

1) Im Wollschweiss finden sich bekanntlich Kali-Seifen, dieselben scheinen ihre Entstehung einem Gehalt an kohlensaurem Kalium zu verdanken, der im Secret der Schweissdrüsen des Schaafs sich findet. Man hat früher angenommen, dass durch dasselbe ein Theil des Wollfetts zerlegt werde. Dio Entstehung der: Seifen erlslärt sich viel leichter durch das Vorkommen freier Fettsäuren im Wollfett, auf welche das kohlensaure Kalium einwirken kann. Doch erscheint es auch nicht undenkbar, dass mit Hülfo der Seifen ein kleiner Theil des Wollfettes emulsionirt wird und dass dann nicht nur dio freien Säuren desselben vollständig neutralisirt, sondern auch die in demselben enthaltenen zusammengesetzten Aother theilweise durch das kohlensanre Kalium zerlegt werden.

3) Landw. Versuchsstat. 17, 15. 